\title{
VARIÁVEIS DE PROCEDIMENTO NA PESQUISA SOBRE CLASSES DE EQUIVALENCIA: CONTRIBUIÇÕES PARA O ESTUDO DO COMPORTAMENTO SIMBOLLICO ${ }^{1}$
}

\section{PROCEDURAL VARIABLES IN EQUIVALENCE CLASSES RESEARCH: CONTRIBUTIONS TO THE STUDY OF SYMBOLIC BEHAVIOR}

\author{
Romariz da Silva Barros, Olavo de Faria Galvão, Ana leda de Faria Brino e \\ Paulo Roney Kilpp Goulart
}

UNIVERSIDADE FEDERAL DO PARÁ, BRASIL

\author{
William Jay McIlvane \\ UNIVERSITY OF MASSACHUSETTS MEDICAL SCHOOL / SHRIVER CENTER, UNITED STATES
}

\begin{abstract}
RESUMO
Os procedimentos tipicamente usados em Análise Experimental do Comportamento para estudar a formação de classes de equivalência podem constituir um modelo experimental para estudar o comportamento simbólico. A formação de classes de equivalência e o comportamento simbólico têm em comum o fato de elementos dissimilares, uma vez arbitrariamente relacionados, se tornaram equivalentes, ou seja, se tornaram substituíveis no que diz respeito ao controle de repertórios a eles associados. Dificuldades para obtenção de classes de equivalência com organismos nãolingüísticos têm levado à especulação de que a formação de classes de equivalência, e possivelmente o comportamento simbólico, seja um fenômeno dependente de funcionamento lingüístico. Alguns trabalhos relatados na literatura, contudo, sugerem que as dificuldades de obtenção de classes de equivalência com organismos não-lingüísticos podem decorrer da falha dos procedimentos para efetivamente treinar as relaçôes de controle planejadas pelo experimentador. O presente trabalho apresenta uma breve revisão de conceitos básicos sobre relações de equivalência, discute algumas das possibilidades de desenvolvimento de controle incoerente em estudos sobre equivalência e aponta brevemente alguns estudos sobre procedimentos alternativos para selecionar relações de controle coerentes com as relações experimentalmente planejadas.
\end{abstract}

Palavras-chave: classes de equivalência, comportamento simbólico, topografia de controle de estímulos, comportamento animal.

\section{ABSTRACT}

Procedures typically used in Experimental Analysis of Behavior for studying equivalence classes formation may constitute an experimental model for studying symbolic behavior. Equivalence classes formation and symbolic behavior are alike in the fact that dissimilar elements, once arbitrarily related, become equivalent, that is, they become substitutable concerning to the control of the repertoires related to them. Difficulties in obtaining equivalence classes with non-linguistic organisms have lead some theorists to speculate that equivalence class formation, and possibly symbolic behavior, is a phenomenon dependent on linguistic functioning. Some studies reported in the literature, however, suggest that difficulties to obtain equivalence classes with non-linguistic organisms may be due to procedural failure to effectively establish stimulus control relations planned by the experimenter. This paper presents a brief review of basic concepts on equivalence relations, discusses some of the possibilities for development of non-coherent stimulus control in equivalence studies, and briefly points to studies of alternative procedures to select stimulus control relations that cohere with the experimentally planned relations.

Key words: equivalence classes, symbolic behavior, stimulus control topography, animal behavior.

1 Romariz da Silva Barros, Olavo de Faria Galvão e Ana Leda de Faria Brino são bolsistas do CNPq. Paulo Roney Kilpp Goulart é bolsista do convênio UFPA/ FADESP/University of Massachusetts/National Institute of Health (USA). William Jay Mcllvane é financiado pelo Center National Institute of Child Health and Human Development (USA) - Grant Number HD 33802. Endereço para correspondência: Romariz Barros, Departamento de Psicologia Experimental, Universidade Federal do Pará, Campus Universitário do Guamá, Rua Augusto Corrêa, 01, CEP 66075-110 - Caixa postal 479 - Belém PA.E-mail: rsb@cpgp.ufpa.br. 
O comportamento simbólico é abordado por diferentes áreas do conhecimento e carece de definição consensual. Apesar disso, é possível afirmar que ao menos parte do que tem sido chamado de comportamento simbólico se caracteriza como comportamento controlado por relações entre estímulos dissimilares arbitrariamente relacionados (símbolos e seus referentes) e substituíveis entre si (ou seja, equivalentes) de tal maneira que o símbolo e seu referente podem exercer a mesma função no controle de repertórios específicos do organismo.

Assim, uma definição possível para o comportamento simbólico pode estar baseada na característica de substitutabilidade que símbolos e seus referentes mantêm entre si, apesar de serem claramente distintos (Bates, 1979). Palavras e outros símbolos, por exemplo, mantêm uma relação de substitutabilidade com os eventos aos quais estão arbitrariamente relacionados (Sidman, 1994 - introdução).

Uma criança que aprendeu a citar pelo nome cada um de seus dois irmãos gêmeos pode, sem nenhum ensino adicional, classificar as peças de vestuário associadas a cada um deles de acordo com os mesmos nomes. O fato de as pessoas e as peças de vestuário, uma vez arbitrariamente associadas, se tornarem substituíveis no controle do repertório de classificação ou nomeação sugere comportamento simbólico.

No contexto da Análise Comportamental, Sidman e seus colaboradores iniciaram, há mais de trinta anos, uma fecunda linha de estudos sobre como estímulos arbitrariamente relacionados podem se tornar substituíveis (Sidman, 1971). Eles nomearam o fenômeno de formação de classes de equivalência (Sidman \& Tailby, 1982). No estudo pioneiro (Sidman, 1971), algumas relações condicionais arbitrárias entre estímulos foram ensinadas e relações não ensinadas, mas esperadas caso os estímulos relacionados condicionalmente tivessem se tornado equi- valentes, foram testadas. Dados positivos foram encontrados nos testes de substitutabilidade. Esses dados estão de acordo com um conceito matemático de relações de equivalência, o qual implica que todos os elementos relacionados por equivalência são intercambiáveis entre si.

O procedimento usualmente utilizado para o estabelecimento de relações condicionais é o procedimento de escolha de acordo com o modelo, que consiste basicamente na apresentação de tentativas em que o participante escolhe entre dois ou mais estímulos de comparação condicionalmente a um estímulo modelo (Carter $\&$ Werner, 1978; Cumming \& Berryman, 1961, 1965). A proposição inicial de Sidman e seus colaboradores consistiu em afirmar que estímulos relacionados condicionalmente, no contexto deste procedimento, se tornam equivalentes.

Apenas a título de exemplo, considere um experimento fictício no qual se ensinariam as seguintes relações arbitrárias a uma criança sem qualquer repertório de leitura. As palavras escritas BRASIL e ARGENTINA (daqui em diante nomeadas de estímulos A1 e A2) seriam arbitrariamente relacionadas às bandeiras respectivamente do Brasil e da Argentina (estímulos B1 e B2). De acordo com o que é usual no procedimento de escolha de acordo com o modelo, cada tentativa iniciaria com a apresentação de uma das palavras à criança como estímulo modelo; uma resposta de observação (usualmente tocar com uma das mãos) à palavra (modelo) seria requerida e, então, as duas bandeiras seriam apresentadas, como estímulos de comparação ${ }^{2}$. A escolha (toque) da bandeira brasileira (mas não da bandeira argentina), dada a palavra BRASIL como modelo, produziria reforçamento (por exemplo, uma guloseima). A escolha da bandeira argentina (mas não da bandeira brasileira), dada a palavra ARGENTINA como modelo, produziria reforçamento. Assim as respectivas palavras e bandeiras poderiam se tornar arbitrariamente relacionadas.

2 Neste exemplo, para tornar a apresentação mais didática, apenas dois estímulos de comparação seriam apresentados. Contudo, é comum o uso de três estímulos de comparação ao invés de dois, como forma de reduzir a probabilidade de que participante faça escolhas exclusivamente baseadas na rejeição da comparação "errada", o que determinaria que nenhuma relação positiva ente o modelo e a comparação correta fosse efetivamente estabelecida. 
As bandeiras, por sua vez, poderiam ser respectivamente relacionadas, como estímulos modelo, às palavras escritas REAL e PESO (estímulos de comparação C1 e C2) por meio do mesmo procedimento. Até aqui, no nosso experimento, as relações $\mathrm{A} 1 \mathrm{~B} 1$ (onde, $\mathrm{A} 1$ = palavra BRASIL e B1 = bandeira do Brasil), A2B2 (onde, A2 = palavra ARGENTINA e B2 = bandeira da Argentina) e $\mathrm{B} 1 \mathrm{C} 1$ (onde, $\mathrm{B} 1$ = bandeira do Brasil e $\mathrm{C} 1$ = palavra REAL), B2C2 (onde, B2 = bandeira da Argentina e C2 = palavra PESO) teriam sido ensinadas, relações estas que podem ser chamadas, para efeitos didáticos, respectivamente de relações $\mathrm{AB}$ e $\mathrm{BC}$.

Ainda considerando o experimento hipotético acima, classes de equivalência seriam constatadas, de acordo com o modelo descritivo de Sidman e seus colaboradores, se recombinações dos elementos positivamente relacionados (A1, B1 e C1, entre si e A2, B2 e C2 entre si) fossem verificadas sem ensino adicional, como por exemplo: 1) as relações B1A1, B2A2, C1B1 e C2B2, que constatam que as relações treinadas comportam a propriedade de simetria, ou seja, a propriedade de reversibilidade; 2) as relações $\mathrm{A} 1 \mathrm{C} 1$ e $\mathrm{A} 2 \mathrm{C} 2$, que constatam que as relações treinadas comportam a propriedade de transitividade, ou seja, estímulos indiretamente relacionados mostram a mesma relação quando diretamente confrontados, e 3) as relações A1A1, A2A2, B1B1, B2B2, $\mathrm{C} 1 \mathrm{C} 1$ e $\mathrm{C} 2 \mathrm{C} 2$, que constatam a propriedade de reflexividade, ou seja, a relação treinada entre os estímulos arbitrários se mostra também entre cada estímulo e ele mesmo. Uma evidência forte da formação de classes de equivalência seria a documentação das relações (ou recombinações) C1A1 e C2A2, que constituem uma constatação das propriedades de simetria e transitividade combinadas. Provavelmente por seu destaque na demonstração de classes de equivalência, dentro deste modelo descritivo, o teste desse tipo de recombinação simétrico-transitiva passou a ser chamado de teste de equivalência.

Em função das convergências de características formais entre o modelo descritivo de Sidman para a formação de classes de equivalência, sucintamente descrito acima, e a definição de comportamento simbólico com a qual estamos lidando aqui, especialmente no que diz respeito à produtividade de relaçōes arbitrárias, ou seja, a constatação de relaçôes não treinadas que consistem em recombinaçôes de relações ensinadas, Sidman (1994) postulou que a formação de classes de equivalência pode constituir um modelo experimental útil para o estudo do comportamento simbólico ou ao menos de seus rudimentos.

Algumas vantagens da adoção deste modelo experimental podem ser citadas: 1) ele está baseado em um procedimento bem estabelecido (o procedimento de escolha de acordo com o modelo) e que é utilizado pela comunidade de pesquisadores de diversas áreas além da Psicologia, como a Neurociência, a Psicofarmacologia e a Farmacologia Comportamental; 2) o procedimento permite a coleta de dados precisos de freqüência de respostas e latências, inclusive com o auxílio de recursos informatizados; 3) o procedimento permite uma ampla variação de categorias de estímulos e respostas a serem usados bem como diferentes protocolos de treino; 4) o modelo experimental parece adequado para a obtenção de dados, produzidos em condiçōes similares, tanto com animais não-humanos quanto com pessoas com desenvolvimento típico e verbalmente hábeis, ou com o desenvolvimento atrasado e repertório verbal rudimentar, o que pode favorecer a produção de conhecimento a respeito da ocorrência do fenômeno em diferentes condições.

De acordo com a proposição teórica de Sidman (1990, 2000), sobre a origem das relações de equivalência, estas são produto direto das contingências de reforçamento, ou seja, decorrem diretamente da interação dos organismos com conjuntos de eventos que estão relacionados (ou são interdependentes) na produção de reforçadores. As classes são inferidas por meio da demonstração da existência de relaçōes de controle intercambiáveis entre os eventos arbitrariamente relacionados (incluindo-se aí estímulos discriminativos, respostas e reforçadores quando específicos em uma classe em oposição a outra ou outras classes) nas contingências de reforçamento. Essas relaçôes se tornam 
efetivas quando o organismo interage com contingências particularmente programadas de maneira a particionar (dividir) em classes específicas uma variedade de elementos originalmente não relacionados entre si.

Assim, é possível inferir a existência de classes de equivalência formadas, por exemplo, entre palavras escritas, faladas e objetos a elas relacionados. Contingências de reforçamento especificamente programadas podem determinar que, uma vez que um desses elementos (uma palavra ou um objeto) está positiva e arbitrariamente correlacionado a um dos elementos de uma contingência de reforcamento (um estímulo condicional ou discriminativo, uma resposta específica ou um reforcador específico), os demais elementos da contingência e outros elementos arbitrariamente relacionados a eles também estarão positivamente relacionados com a produção de reforços, mesmo que nunca antes tenham sido relacionados diretamente.

Voltando ao nosso experimento fictício, para ficar mais claro, poder-se-ia organizar contingências de maneira que os seis elementos citados (as palavras BRASIL, PESO, REAL e ARGENTINA e as bandeiras do Brasil e da Argentina) seriam classificados por uma criança de diferentes maneiras. Por exemplo, os seis elementos poderiam ser divididos em duas classes: palavras $(\mathrm{n}=4)$ versus bandeiras $(\mathrm{n}=2)$; ou em três classes: palavras com 4 letras $(\mathrm{n}=2)$, palavras com mais de 4 letras ( $n=2)$ e bandeiras $(n=2)$, ou ainda, a depender de algum repertório de leitura: nomes de países ( $\mathrm{n}=2)$, nomes de moedas ( $\mathrm{n}=2$ ) e bandeiras ( $\mathrm{n}=2)$.

Contudo, no experimento hipotético acima, nenhum desses "critérios" de classificação ou agrupamento teria sido adotado. De fato, no estudo fictício acima, o experimentador teria arbitrariamente programado as contingências de reforçamento de maneira a determinar que a palavra BRASIL, a bandeira brasileira e a palavra REAL estivessem positivamente correlacionadas de maneira que a seleção de um desses elementos, dado qualquer outro da mesma classe como modelo, culminaria na produção de reforçadores, mesmo que uma ou outra combinação em particular nunca tivesse sido antes apresentada. $\mathrm{O}$ mesmo ocorreria com a palavra ARGENTINA, a bandeira argentina e a palavra PESO. Isso seria feito inicialmente associando diretamente a palavra BRASIL à bandeira do Brasil e, então, associando-se esta à palavra REAL. Ao mesmo tempo, a palavra ARGENTINA seria diretamente associada à bandeira da Argentina e esta, por sua vez, à palavra PESO. As demais re-combinaçôes poderiam ser testadas.

Portanto, o que se estabeleceria concretamente seriam contingências que determinariam a substituibilidade ou equivalência entre os elementos de uma mesma classe, de tal forma que quando um deles está positivamente correlacionado com a produção de reforços, os demais também estão. As classes de equivalência poderiam então ser construídas no repertório da criança, como produto da sua interação com esse arranjo de contingências e seriam inferidas se seu desempenho, ao fazer escolhas condicionais frente ao modelo, fosse consistente com a partição do conjunto de seis elementos em dois conjuntos de três (Brasil vs. Argentina), conforme acima descrito.

Recentemente, Sidman $(1994,2000)$ propôs que as respostas e as consequiências que integram as contingências, quando específicas para cada classe, também podem ser consideradas como parte do conjunto de elementos que, uma vez relacionados, podem se tornar substituíveis ou recombináveis entre si, integrando, portanto as classes de equivalência. Considerando o exemplo acima, uma resposta específica para cada uma das duas classes desenvolvidas poderia ser selecionada pelas contingências de reforcamento. Por exemplo, para os estímulos da classe "Brasill" a resposta seria tocar cinco vezes o estímulo (razão fixa 5) dentro de um intervalo máximo de dois segundos (o que seria aqui considerado como Resposta 1 ou simplesmente R1). Para os estímulos da classe "Argentina”, a resposta selecionada seria tocar duas vezes o estímulo com espaçamento mínimo entre respostas de 3 segundos - DRL 3 (Resposta 2 ou simplesmente R2). Também conseqüências específicas de cada classe poderiam ser providas através das contingências. Por exemplo, jujuba (Conseqüência 1) para a classe "Brasil" e bala de coco (Conseqüência 2) para a classe "Argentina”. Pelo modelo descritivo, as 
respostas e as conseqüências poderiam passar a integrar as classes. Essa extensão do modelo descritivo de classes de equivalência nos permite inclusive postular que essas classes podem ser construídas por meio de contingências mais simples do que a contingência de quatro termos anteriormente exemplificada.

Assim, teoricamente classes de equivalência poderiam ser obtidas, por exemplo, via contingências de três termos (estímulo discriminativo, resposta e reforço) (ver Sidman, 2000). Nessa perspectiva, um arranjo de contingências no qual dois estímulos discriminativos diferentes (por exemplo A1 e A2) que controlariam duas respostas diferentes (por exemplo, respectivamente R1 e R2), mantidas por consequiências específicas (por exemplo, respectivamente Conseq. 1 e Conseq. 2) pode produzir duas classes de equivalência (A1-R1-Conseq.1 e A2-R2Conseq.2). Novos estímulos discriminativos (por exemplo, B1 e B2) respectivamente associados às respostas e conseqüências (R1-Conseq.1 e R2-Conseq.2) podem passar a fazer parte das classes (então, B1-A1-R1-Conseq.1 e B2-A2-R2-Conseq.2). Uma maneira de averiguar isso seria verificar se as realações entre estímulos A1-B1 e A2B2 emergem sem treino. A ampliação do modelo descritivo de classes de equivalência, ao introduzir a possibilidade de utilização de procedimentos mais simplificados (ver Sidman, 2000), abre novas perspectivas para o desenvolvimento de modelos para o estudo do comportamento simbólico em organismos não-humanos ou em humanos com desenvolvimento severamente atrasado.

Ocorre que alguns estudos sobre classes de equivalência, em que sujeitos humanos com severas limitações de repertório lingüístico ou sujeitos não-humanos foram submetidos aos procedimentos tradicionais de treino e teste, não apresentaram evidências - ou apresentaram indícios insuficientemente convincentes - de formação de classes, ao passo que desempenhos consistentes com os previstos para a demonstração de equivalência são corriqueiramente encontrados quando participantes humanos com funcionamento lingüístico típico são submetidos àqueles procedimentos. Devany, Hayes e Nelson (1986) e Sidman,
Rauzin, Lazar, Cunninghan, Tailby e Carrigan (1982) desenvolveram estudos largamente citados como evidências da dificuldade de obtenção de classes de equivalência com participantes com baixo funcionamento lingüístico (crianças e adolescentes com desenvolvimento severa e atipicamente atrasado, por exemplo) ou com animais. Alguns teóricos então passaram a especular que o fenômeno da formação das classes de equivalência seria um produto do funcionamento lingüístico ou, mais especificamente, produto de repertórios de nomeação (Dugdale \& Lowe, 1990; Hayes, 1991; Horne \& Lowe, 1996).

Muitos dos trabalhos relatados na literatura objetivando a demonstração de relações emergentes por sujeitos não-humanos não produziram evidências de formação de classes em testes das propriedades emergentes de reflexividade, simetria e transitividade, ou apontaram evidências parciais de uma ou outra dessas propriedades, mas não de todas (D’Amato, Salmon, Loukas, \& Tomie, 1985; Dugdale \& Lowe, 2000; Kuno, Kitadate, \& Iwamoto, 1994; Lionello-DeNolf \& Urcuioli, 2002; Tomonaga, Matsuzawa, Fugita, \& Iamamoto, 1991; Urcuioli \& Zental, 1993), ou obtiveram achados sugestivos de formação de classes, mas com procedimentos diferenciados que dificultavam, em certa medida, a comparação direta com outros trabalhos (Cerutti \& Rumbaugh, 1993; Manabe, Kawashima, \& Staddon, 1995; McIntire, Cleary, \& Thompson, 1987; Vaughan, 1988), especialmente quando ainda não se cogitava a inclusão de respostas e consequiências como membros de classes de equivalência.

Contudo, com a ampliação do modelo descritivo de classes de equivalência, isto é, com a inclusão de reforçad ores e respostas como potenciais membros de classes de equivalência, estudos como o de McIntire et al. (1987) e Vaughan (1988) voltaram à discussão porque parecem mais compatíveis com a definição ampliada de classes de equivalência.

No estudo de McIntire et al. (1987), por exemplo, foram obtidos resultados positivos em testes de reflexividade, simetria e transitividade com macacos Rhesus (Macaca mulata) como sujeitos e cores como estímulos. O 
procedimento incluiu inicialmente o treino de uma resposta diferencial para cada classe potencial de estímulos. Assim, após treino discriminativo, cada um de três estímulos (A1, B1 e C1, por exemplo) controlava a emissão de uma determinada resposta $\mathrm{R} 1$ pelo sujeito e outros três estímulos (A2, B2 e C2) controlavam a emissão de outra resposta R2. Só então, foi efetuado o treino de relações condicionais por meio do procedimento de escolha de acordo com o modelo, sempre associando estímulos já previamente relacionados a uma das respostas específicas. Por exemplo, quando o modelo era o estímulo A1, era requerida a resposta específica R1 e então os estímulos B1 e B2 eram apresentados. Então, somente a emissão da resposta R1 ao estímulo B1 produzia reforçamento. Quando o modelo era o estímulo $\mathrm{A} 2$, a resposta específica $\mathrm{R} 2$ era requerida e, então, os estímulos B1 e B2 eram apresentados. Somente a emissão da resposta R2 ao estímulo B2 produzia reforçamento. As contingências estabelecidas, portanto, selecionavam não apenas a escolha da comparação definida como correta, frente a cada modelo, mas também a topografia de resposta definida como correta. O mesmo procedimento foi adotado no treino BC. Nos testes de propriedades emergentes, os sujeitos sempre selecionavam entre os estímulos de comparação aquele que controlava a mesma topografia de reposta emitida ao modelo. Por exemplo, no teste CA, dado que os sujeitos tinham emitido a resposta $\mathrm{R} 1$ ao modelo $\mathrm{C} 1$, eles selecionavam $\mathrm{A} 1$ ao invés de A2, emitindo a resposta R1. A mediação de respostas diferenciais na relação modelo-comparação no estudo de McIntire et al. (1987) foi considerada inicialmente um artefato que produziu não mais que aparentes classes de equivalência com sujeitos não-humanos (Hayes, 1989). Contudo, considerando que as repostas podem ser consideradas simplesmente como membros adicionais das classes (A1-B1-C1-R1 e A2-B2-C2-R2), é possível que os dados obtidos naquele estudo possam representar uma interessante demonstração de uma predição teórica do modelo expandido de classes de equivalência mais recentemente proposto por Sidman (ver Sidman, 1994, Capítulo 5). Replicaçôes deste estudo devem ser efetuadas, para que se possa aprofundar sua discussão.

Outro interessante estudo foi publicado por Vaughan (1988). Pombos foram utilizados como sujeitos em um procedimento de reversōes de discriminaçōes simples sucessivas com dois conjuntos de 20 estímulos. Após treino discriminativo, dentre 40 slides de árvores, 20 controlavam precisamente a emissão de respostas de bicar e os demais 20 estímulos controlavam outras respostas que não a de bicar. Os animais, portanto, bicavam, dentre os 40 estímulos, os 20 que funcionavam $S+$ e não bicavam os 20 que funcionavam como $S$-. Após a aquisição das discriminações, a função dos estímulos era invertida. Após várias reversões das funções dos estímulos dos dois conjuntos, os animais passaram a mudar consistentemente seu responder frente a todos os estímulos dos conjuntos, após o contato com as conseqüências produzidas pelas respostas a alguns, mas não todos, estímulos de cada conjunto, no início das sessōes.

Estes resultados foram aceitos como uma convincente evidência de formação de classes de estímulos funcionalmente equivalentes em não-humanos. Apesar de as classes de estímulos observadas por Vaughan (1988) compartilharem certas características com as classes de estímulos produzidas no contexto de escolha de acordo com o modelo, como a substitutabilidade funcional de seus membros no controle do comportamento, tradicionalmente era requerido que fossem realizados testes para verificação das propriedades emergentes (reflexividade, simetria e transitividade) para se considerar que classes de equivalência tivessem sido obtidas, de acordo com o modelo descritivo amplamente aceito e aqui anteriormente apresentado.

Contudo, a despeito das dificuldades metodológicas para a verificação empírica da equivalência entre classes funcionais e classes de equivalência, tem sido sugerido, mais recentemente, que classes funcionais e classes de equivalência envolvem os mesmos processos comportamentais, estando a distinção estabelecida entre ambos os fenômenos, portanto, restrita aos procedimentos dos quais se originam. Dados que confirmam essa posição começam a ser encontrados, por exemplo, por Kastak, Schusterman e 
Kastak (2001) com leões marinhos (ver também CohenAlmeida, 1993; de Rose, Mcllvane, Dube, \& Stoddard, 1988; Dube, McDonald, \& Mcllvane, 1991; Dube, McIlvane, Callahan, \& Stoddard, 1993; Galvão, 1993; Sidman, 1994, pp. 416-421).

Além disso, a formação de classes funcionais vem sendo considerada um fenômeno fundamental para a investigação dos pré-requisitos comportamentais necessários para a obtenção de relações de equivalência (Dube, McIlvane, Callahan, \& Stoddard, 1993; Kastak et al., 2001).

No estudo desenvolvido por Kastak et al. (2001), acima mencionado, inicialmente os dados de formação de classes funcionais por meio do procedimento de reversões repetidas de discriminaçôes simples (obtidos por Vaughan [1988] com pombos) foram replicados com dois leões marinhos. Em seguida, os pesquisadores encontraram que as mesmas classes produzidas por meio do procedimento de reversões repetidas de discriminaçôes simples, podiam ser observadas, sem treino adicional, no contexto do procedimento de escolha de acordo com o modelo, sugerindo que classes funcionais e classes de equivalência envolvem os mesmos processos comportamentais. Evidências adicionais disso foram encontradas quando novos estímulos, incluídos nas classes de equivalência, por meio do procedimento de escolha de acordo com o modelo, também passaram a integrar as classes funcionais, sem treino adicional, no procedimento de reversões de discriminações simples, como também novos estímulos introduzidos nas classes funcionais, por meio do procedimento de reversões repetidas de discriminaçōes simples, passaram a integrar as classes de equivalência sem treino adicional, no procedimento de escolha de acordo com o modelo.

Também Schusterman e Kastak (1993) encontraram fortes evidências da formação de classes de estímulos com sujeitos não-humanos (desta vez com o procedimento padrão para a formação de classes de equivalência e usando um leão-marinho como sujeito). Nesse estudo, dois leões marinhos foram expostos a vários exemplares de tarefas de escolha de acordo com o modelo, tanto por identidade (ver Kastak \& Schusterman, 1994) como arbitrárias. Após o treino de 12 diferentes exemplares de relaçôes $A B$, testes de simetria $B A$ foram conduzidos. Não foram encontradas evidências convincentes de propriedade de simetria até este ponto. Contudo, as relações simétricas BA, não emergentes, foram diretamente treinadas e, então, foram também treinadas 12 relações condicionais BC. Testes de simetria CB e de transitividade AC foram conduzidos, obtendo-se fortes evidências da emergência dessas propriedades. Também foram encontradas fortes evidências da formação de classes de equivalência em testes das relaçóes $\mathrm{CA}$ após treino $\mathrm{AB}$ e $\mathrm{BC}$ com 18 novos conjuntos de estímulos.

Estes achados com sujeitos não-humanos, somados a algumas evidências de que mesmo crianças com repertório lingüístico muito pobre exibem classes de equivalência (Carr, Wilkinson, Blackman, \& McIlvane, 2000), indicam que a posição dos teóricos que postulam a linguagem ou a nomeação como fonte das classes de equivalência é prematura. É possível que as dificuldades para encontrar classes de equivalência em organismos não lingüísticos decorra de preparação e adaptação insuficiente de procedimentos para esse tipo de sujeitos.

Alguns estudos têm mostrado que sujeitos não-humanos, por exemplo, podem desenvolver relações de controle de estímulo sutilmente diferentes das relações planejadas pelo experimentador, quando submetidos a procedimentos padrão de escolha de acordo com o modelo (Iversen, 1997; Iversen, Sidman, \& Carrigan, 1986; Lionello \& Urcuioli, 1998). Nesses estudos foi demonstrado que, além dos aspectos do estímulo originalmente planejados pelo experimentador para controlar o desempenho dos sujeitos (cores, linhas etc), também a posição em que esses estímulos eram apresentados adquiriu controle sobre o desempenho dos sujeitos. Em parte, essa divergência entre topografias de controle de estímulos planejadas e efetivamente desenvolvidas pode explicar a dificuldade de formação de classes de equivalência com sujeitos não-humanos no procedimento padrão de escolha de acordo com o modelo. 
Dube e Mcllvane (1996), fazendo uso da noção de topografia de controle de estímulos (Ray, 1969), argumentaram que múltiplas relações de controle (algumas vezes conflitantes com as relações planejadas pelo experimentador) podem coexistir quando um sujeito desempenha tarefas de escolha de acordo com o modelo. A noção de topografia de controle de estímulos é análoga à noção de topografia de respostas. Quando dizemos que uma classe de respostas está sendo reforçada, estamos afirmando que uma variedade de respostas topograficamente diferentes, mas que produzem o mesmo efeito, está sendo reforçada. A noção de topografia de controle de estímulos consiste em decompor uma medida global de desempenho numa discriminação condicional, por exemplo, em seus diversos componentes, ou seja, consiste em identificar as diferentes relações de controle que podem estar sendo mantidas pelas contingências de reforçamento.

Apenas a título de exemplo, consideremos o treino de duas relações condicionais arbitrárias, entre os estímulos A1 e B1 e entre os estímulos A2 e B2 por meio do procedimento padrão de escolha de acordo com o modelo com três chaves de resposta alinhadas horizontalmente e com os estímulos modelo (neste caso, A1 e A2) sempre apresentados na janela central e os estímulos de comparação sempre apresentados nas janelas laterais. O plano experimental, neste caso, prescreveria que as contingências selecionassem topografias de controle tais como: "dado o modelo A1, o participante seleciona a comparação B1" e "dado o modelo A2, o participante seleciona a comparação B2”. Num bloco de 100 tentativas, em que 50 são do tipo A1B1 e 50 são do tipo A2B2 (com as escolhas definidas como corretas apresentadas igual número de vezes 25 - nas posições laterais da esquerda e da direita), cada uma das duas topografias de controle de estímulos acima citadas apareceria com freqüência igual a 50 e, neste caso, a precisão do desempenho do participante seria de $100 \%$ de acertos.

Contudo, se o participante mostrasse as seguintes topografias de controle "dado o modelo A1, selecionar a comparação B1" e "dado o modelo A2, selecionar a chave da esquerda", a precisão final do desempenho cairia para $75 \%$. Todas as tentativas do tipo A1B1 seriam concluídas com reforçamento, o que produziria $50 \%$ da precisão global do bloco de tentativas, mas apenas metade das tentativas do tipo A2B2 seriam concluídas com reforçamento (considerando que, em metade das tentativas A2B2, a escolha definida como correta estaria disponível na posição esquerda) o que produziria $50 \%$ de precisão nas tentativas do tipo A2B2 e 25\% de precisão global no bloco de tentativas. Isso determinaria que a topografia de controle de estímulos “se A1, então B1" estaria sendo reforçada em um esquema de reforçamento contínuo e a topografia "se A2, então esquerda", apesar de conflitante com o planejamento experimental, estaria sendo reforçada em VR2. Esse arranjo de contingências, portanto, poderia ser suficiente para manter ambas as topografias de controle. O desempenho selecionado por essas contingências seria, portanto, neste caso, divergente do desempenho planejado pelo experimentador. $\mathrm{O}$ fato de a relação condicional A2B2 não estar sendo reforçada (mas sim a relação A2-esquerda) certamente produziria resultados inconsistentes com a formação de classes de equivalência em testes posteriores, como, por exemplo, testes de simetria BA.

Para o pesquisador ou educador, neste exemplo, seria de grande valor conhecer a fonte da imprecisão no desempenho do participante, notadamente o controle não programado pela posição esquerda nas tentativas do tipo A2B2. Esse seria o primeiro passo, fundamental para a implementação de mudanças de sucesso no procedimento. Explicaçôes apoiadas meramente em alegações de incapacidades dos participantes não contribuiriam significativamente.

Mesmo que a precisão do desempenho do participante, no exemplo acima, fosse de $100 \%$ em ambas as relações A1B1 e A2B2, ainda assim seria possível que o treino tivesse gerado relaçôes de controle incoerentes com aquelas planejadas. Johnson e Sidman (1993) argumentaram que mesmo quando um participante seleciona, com altíssima precisão, a comparação arbitrária nominalmente definida pelo experimentador como correta, dado o estí- 
mulo modelo, relações de controle diferentes das planejadas podem estar implicadas (ver também Carrigan \& Sidman, 1992; Sidman, 1987, 1994). As respostas de escolha dos participantes podem estar sendo controladas por aspectos do $S_{+}$(comparação definida pelo experimentador como correta), o que nos permitiria dizer que as contingências teriam selecionado relações de controle por seleção do $S_{+}$. Contudo, as respostas de escolha, de maneira não programada pelo experimentador, poderiam estar sendo controladas por uma relação de rejeição ao S- (estímulo definido pelo experimentador como escolha errada). Neste caso, o desempenho do participante estaria sob controle de aspectos do $S$ - e o participante então rejeitaria o S-, apontando ou tocando o $S_{+}$. Em ambos os casos, o desempenho resultante seria, aparentemente, consistente com as contingências programadas e seria impossível distinguir as duas topografias de controle apenas observando o participante desempenhar a tarefa.

No caso de o controle do comportamento estar sendo exercido pelo estímulo definido como negativo (controle por rejeição), o sujeito, mesmo tendo respondido em alta precisão durante o treino das relações de linha de base, provavelmente não apresentaria todos os desempenhos emergentes previstos em testes para verificação de classes de equivalência, pois estímulos potencialmente integrantes das classes de equivalência, de acordo com o plano experimental, não estariam de fato positivamente relacionados na contingência (do ponto de vista do participante), fato que é necessário para a obtenção das recombinaçōes que evidenciariam a formação de classes de equivalência.

Divergências no controle de estímulos em relação às demandas dos testes de relações de equivalência, como estas previstas por Johnson e Sidman (1993), poderiam ser evitadas se as contingências de treino fossem arranjadas de forma a restringir o desenvolvimento de relações de controle às relações planejadas. Esse problema, bem como algumas propostas para sua resolução, tem sido abordado sob o título de "Teoria da Coerência de Topografia de Controle de Estímulos" (Dube \& McIlvane, 1996; McIlvane, Serna, Dube, \& Stromer, 2000). De acordo com essa teoria relativamente simples, qualquer afirmação sobre o desempenho de participantes em tarefas de escolha de acordo com o modelo tem que estar baseada na coerência entre o que o experimentador ou educador considera que foi ensinado e o desempenho realmente desenvolvido ao longo do procedimento de ensino. Procedimentos e arranjos experimentais especiais podem ser necessários para garantir que o comportamento de sujeitos fique sob controle das propriedades relevantes da tarefa (do ponto de vista do experimentador).

Mais uma vez, é possível que a incoerência entre desempenho planejado e efetivamente desenvolvido explique parte dos problemas para obtenção de classes de equivalência em sujeitos não-humanos.

Considerando os cuidados para evitar as possibilidades de digressōes de controle de estímulo acima mencionadas, tem se explorado a possibilidade de desenvolvimento de classes de estímulos em sujeitos não-humanos da espécie Cebus apella (macaco-prego) em um programa de pesquisa intitulado "Escola Experimental de Primatas" (ver Barros, Galvão, \& Mcllvane, 2003; Galvão, Barros, Rocha, Mendonça, \& Goulart, 2002). A proposição central do programa de pesquisa é a promoção de coerência de topografia de controle de estímulos para exploração do potencial dos participantes para a aquisição de repertórios complexos como a formação de classes de equivalência. $\mathrm{O}$ estudo pode contribuir para a o desenvolvimento de procedimentos para exploração do potencial simbólico de sujeitos não-humanos e participantes humanos com desenvolvimento severamente atrasado.

Algumas alterações de procedimento em relação aos procedimentos convencionais foram introduzidas como forma de evitar incoerências de controle de estímulos classicamente relatadas na pesquisa com sujeitos não-humanos, como o controle pela posição dos estímulos.

Sidman (1994) e Iversen et al. (1986), por exemplo, chamaram a atenção para o fato de que a correlação entre a posição dos estímulos (modelo na janela central e comparações em janelas laterais), tradicionalmente adotada no procedimento de escolha de acordo com o mo- 
delo, pode determinar que a posição dos estímulos controle o desempenho dos sujeitos juntamente com os elementos nominalmente programados pelo experimentador para desenvolver controle. Em função disso, temos utilizado uma variação do procedimento de escolha de acordo com o modelo na qual tanto os estímulos modelo quanto os estímulos de comparação são apresentados randomicamente em 9 posições distribuídas em uma matriz $3 \times 3$. Essa pequena variação no procedimento elimina a correlação entre a posição dos estímulos e sua função enquanto modelo e comparação, restringindo a possibilidade de controle aos aspectos dos estímulos propriamente ditos.

Goulart, Mendonça, Barros, Galvão e McIlvane, (no prelo) utilizaram, pioneiramente com sujeitos nãohumanos, o procedimento de "comparação vazio" (McIlvane, Kledaras, Munson, King, de Rose, \& Stoddard, 1987) para identificação de relações de controle em discriminaçôes simples. O procedimento também tem sido pioneiramente usado na Escola Experimental de Primatas para a avaliação de relações de controle em discriminações condicionais com macacos Cebus apella e com o procedimento de escolha de acordo com o modelo. O procedimento básico consiste na apresentação de tentativas de sonda em que ora se omite o $S_{+}$, apresentando o $S_{-}$e uma janela vazia, ora se omite o $S$-, apresentando o $S+$ e uma janela vazia. Nas tentativas em que o $S+$ e a janela vazia são apresentados, respostas consistentes ao $S+$ são consideradas evidências de controle por $S_{+}$(ou controle por seleção). Nas tentativas em que o $S$ - e a janela vazia são apresentados, respostas consistentes à janela vazia são consideradas evidências de controle por $\mathrm{S}$ - (ou controle por rejeição). A utilização desse procedimento tem contribuído para a identificação de características sutis do repertório discriminativo dos sujeitos, que são impossíveis de detectar apenas com base na precisão bruta do desempenho dos blocos de tentativas. O mesmo procedimento tem sido utilizado em blocos de ensino, para garantir que relações de controle por seleção e/ou por rejeição (controle misto) sejam selecionadas pelas contingências, mesmo quando o procedimento de escolha de acordo com o modelo com dupla escolha é utilizado.

Adicionalmente, com o objetivo de evitar rompimento das discriminações treinadas quando testes de relações emergentes são introduzidos, Barros, Galvão e McIlvane (2002) e Galvão, Barros, Brandão, Lavratti, Santos, Brino, Dube e McIlvane (no prelo), usaram sessões de testes similares às de treino, com mesmo número e proporção dos diferentes tipos de tentativas, mesma probabilidade de reforçamento e inclusão de sondas sem reforçamento ou com reforçamento intermitente, alternadamente, em sessões de linha de base e de teste, para permitir testes sucessivos sem efeito de queda na precisão do desempenho nas tentativas de teste (ver também Galvão, Calcagno, \& Sidman, 1992). Os resultados em geral têm indicado efeitos positivos desse conjunto de cuidados, com a obtenção, em larga escala, de identidade generalizada.

Na Escola Experimental de Primatas vem sendo implementada, portanto, uma abordagem ampla para a melhoria da coerência de topografia de controle de estímulos em procedimentos de treino discriminativo. Essa abordagem reflete um passo importante em direção ao desenvolvimento de uma tecnologia comportamental abrangente e confiável para lidar com a questão da coerência de topografia de controle de estímulos, que permita desde uma identificação mais precisa das topografias de controle de estímulos ideais para a demonstração dos desempenhos esperados, até o estabelecimento de topografias de controle que sejam o mais semelhantes possível àquelas. Os avanços atuais ainda são tímidos em relação ao que poderia ser atingido sob condições ideais. Por exemplo, os métodos atuais de modelagem de controle de estímulos, com vistas a atingir um determinado desempenho complexo, como discriminaçóes condicionais arbitrárias, têm que ser feitos de maneira estática: cada passo sucessivo na modelagem tem sido construído individualmente e a sucessão de passos vem sendo construída em blocos separados de tentativas. Em breve, essa metodologia poderá ser aperfeiçoada, tornando a modelagem de controle de estímulos um processo mais dinâmico, no qual, por exemplo, as mudanças 
nos estímulos em um procedimento de modelagem do estímulo modelo (Zygmont, Lazar, Dube, \& Mcllvane, 1992) serão feitas mais fluentemente e considerando diversas variáveis do desempenho dos sujeitos (e.g. precisão, latência de resposta etc). A tecnologia comportamental necessária para dar suporte a tais avanços está razoavelmente bem desenvolvida e seria de grande valor se essa tecnologia fosse traduzida via desenvolvimento de um algoritmo efetivo que permitisse sua implementação computadorizada. Pelo menos um projeto nessa direção encontra-se atualmente em andamento (Richard Serna, comunicação pessoal), através do qual um software está sendo desenvolvido para auxiliar no seqüenciamento de arranjos de contingências para a construção de repertórios complexos, conforme acima sugerido. Essa tecnologia poderá estar disponível para esse campo de pesquisa e atuação profissional em um futuro muito próximo.

Até mesmo técnicas mais avançadas para atingir coerência de topografia de controle de estímulos podem estar dentro de alcance. Aplicações da análise de detecção de sinais (Nevin, 1969) podem permitir análise sistemática da sensibilidade corrente do desempenho às contingências, incluindo análise de respostas desviantes que podem concorrer com a aquisição do repertório planejado (cf. Davison \& Nevin, 1999). Também merecedoras de estudos adicionais são as técnicas para distinção entre topografia de controle desejáveis das não desejáveis. Um exemplo desse procedimento de "filtragem de topografias" pode ser encontrado em um artigo recentemente publicado por Mcllvane, Kledaras, Callahan e Dube (2002). Também o conceito geral e os fundamentos teóricos de uma metodologia para melhorar coerência de topografia de controle de estímulos foram recentemente discutidos em um trabalho publicado por Mcllvane e Dube (2003).

Por fim, longe de pretender esgotar a discussão sobre o avanço de técnicas e de tecnologia para o desenvolvimento de relaçôes de controle do comportamento dos participantes de maneira coerente com o ponto de vista do programador de contingências (seja um pesquisador ou um professor, numa situação aplicada em que a construção de repertório complexo seja necessária), espe- ra-se que o presente texto tenha despertado o interesse do leitor para o tema, ao mesmo tempo em que se aponta alguma literatura relevante para posterior estudo. O avanço desse campo de estudo produzirá importantes contribuições para a compreensão de questôes relacionadas ao comportamento simbólico e para a intervenção sobre esse tipo de comportamento.

\section{REFERÊNCIAS}

Barros, R. S., Galvão, O. F., \& Mcllvane, W. J. (2002). Generalized identity matching-to-sample in Cebus apella. Psychological Record, 52, 441-460.

Barros, R. S., Galvão, O. F., \& McIlvane, W. J. (2003). The search for relational learning capacity in Cebusapella: A programmed educational approach. In S. Soraci Jr. \& K. Murata-Soraci (Eds). Visual information processing, (pp. 223-245). Connecticut, MA: Praeger.

Bates, E. (1979). The emergence of symbols: Cognition and communication in infancy. New York, NY: Academic Press.

Carr, D., Wilkinson, K. M., Blackman, D. E., \& Mcllvane, W. J. (2000). Equivalence classes in individuals with minimal verbal repertoires. Journal of the Experimental Analysis of Behavior, 74, 101-114.

Carrigan, P. F., \& Sidman, M. (1992). Conditional discrimination and equivalence relations: A theoretical analysis of control by negative stimuli. Journal of the Experimental Analysis of Behavior, 58, 183-204.

Carter, D. E., \& Werner, T. S. (1978). Complex learning and information processing by pigeons: A critical analysis. Journal of the Experimental Analysis of Behavior, 29, 565-601.

Cerutti, D. T., \& Rumbaugh, D. M. (1993). Stimulus relations in comparative primate perspective. The Psycological Record, 43, 811-821.

Cohen-Almeida, D. (1993). Simple discrimination tests for transfer of functional class membership. Dissertação de mestrado. Boston, MA. Northeastern University.

Cumming, W. W., \& Berryman, R. (1961). Some data on matching behavior in the pigeon. Journal of the Experimental Analysis of Behavior, 4, 281-284.

Cumming, W. W., \& Berryman, R. (1965). The complex discriminated operant: Studies of matching-tosample and related problems. In D. I. Mostofsky (Org.), Stimulus generalization (pp. 284-330). Stanford, CA: Stanford University Press.

D’Amato, M. R., Salmon, D. P., Loukas, E., \& Tomie, A. (1985). Symmetry and transitivity of conditional relations in monkeys (Cebusapella) and pigeons (Columba livia). Journal of Experimental Analysis of Behavior, 44 , $35-47$. 
Davison, M., \& Nevin, J. A. (1999). Stimuli, reiforcers, and behavior: An integration. Journal of the Experimental Analysis of Behavior, 71, 439-482.

de Rose, J. C., McIlvane, W. J., Dube, W. V., \& Stoddard, L. T. (1988). Stimulus class formation and functional equivalence in moderately retarded individuals' conditional discrimination. Behavioural Processes, 17, 167175.

Devany, J. M., Hayes, S. C., \& Nelson, R. O. (1986). Equivalence class formation in language-able and language-disabled children. Journal of the Experimental Analysis of Behavior, 46, 243-257.

Dube, W. V., \& McIlvane, W. J. (1996). Some implications of a stimulus control topography analysis for emergent stimulus classes (pp. 197-218). In T. R. Zentall \& P. M. Smeets (Orgs.), Stimulus class formation in humans and animals (pp. 197-218). North Holland: Elsevier.

Dube, W. V., McDonald, S. J., \& Mcllvane, W. J. (1991). A note on the relationship between equivalence class and funtional stimulus classes. Experimental Analysis of Human Behavior Bulletin, 9, 7-11.

Dube, W. V., Mcllvane, W. J., Callahan, T. D., \& Stoddard, L. T. (1993). The search for stimulus equivalence in nonverbal organisms. The Psychological Record, 42, 761-778.

Dugdale, N. A., \& Lowe, C. F. (1990). Naming and stimulus equivalence. In D. E. Blackman \& H. Lejeune (Orgs.). Behaviour analysis in theory and practice: Contributions and controversies (pp. 115-138). Brighton, U.K.: Lawrence Erlbaum Associates.

Dugdale, N. A., \& Lowe, C. F. (2000). Testing for symmetry in the conditional discriminations of languagetrained chimpanzees. Journal of the Experimental Analysis of Behavior, 73, 5-22.

Galvão, O. F. (1993). Classes funcionais e equivalência de estímulos. Psicologia: Teoria e Pesquisa, 9, 547-554.

Galvão, O. F., Barros, R. S., Brandão, S., Lavratti, C. M., Santos, J. R., Brino, A. L. F., Dube, W. V., \& McIlvane, W. J. (no prelo). Extent and limits of the matching concept in Cebus apella: A matter of experimental control? The Psychological Record.

Galvão, O. F., Barros, R. S., Rocha, A. C., Mendonça, M. B., \& Goulart, P. R. K. (2002). Escola Experimental de Primatas. Estudos de Psicologia, 7, 361-370.

Galvão, O. F., Calcagno, S., \& Sidman, M. (1992). Testing for emergent performance in extinction. Experimental Analysis of Human Behavior Bulletin, 10, 18-20.

Goulart, P. R. K., Mendonça, M. B., Barros, R. S., Galvão, O. F., \& McIlvane, W. J. (submetido). A note on select and reject controlling relations in the simple discrimination of capuchin monkeys (Cebus apella). Journal of Experimental Psychology: Animal Behavior Processes.

Hayes, S. C. (1989). Nonhumans have not yet shown stimulus equivalence. Journal of the Experimental Analysis of Behavior, 51, 385-392.

Hayes, S. C. (1991). A relational control theory of stimulus equivalence. In L. J. Hayes e P. N. Chase (Orgs.). Dialogues on verbal behavior (pp. 19-40). Reno, NV: Context Press.

Horne, P.J., \& Lowe, C.F. (1996). On the origins of naming and other symbolic behavior. Journal of the Experimental Analysis of Behavior, 65, 185-241.

Iversen, I. H. (1997). Matching-to-sample performance in rats: A case of mistaken identity? Journal of the Experimental Analysis of Behavior, 45, 297-304.

Iversen, I. H., Sidman, M., \& Carrigan, P. (1986). Stimulus definition in conditional discriminations. Journal of the Experimental Analysis of Behavior, 45, 297 304.

Johnson, C., \& Sidman, M. (1993). Conditional discriminations and equivalence relations: Control by negative stimuli. Journal of the Experimental Analysis of Behavior, 59, 333-347.

Kastak, C. R., Schusterman, K. R., \& Kastak, D. (2001). Equivalence classification by California sea lions using class-specific reinforcers. Journal of the Experimental Analysis of Behavior, 76, 131-158.

Kastak, D., \& Schusterman, R. J. (1994). Transfer of visual identity matching-to-sample in two California sea lions (Zalophus californianus). Animal Learning \& Behavior, 22, 427-435.

Kuno, H., Kitadate, T., \& Iwamoto, T. (1994). Formation of transitivity in conditional matching to sample by pigeons. Journal of the Experimental Analysis of Behavior 2 62, 399-408.

Lionello, K. M., \& Urcuioli, P. J. (1998). Control by location in pigeons matching-to-sample. Journal of the Experimental Analysis of Behavior, 70, 235-251.

Lionello-DeNolf, K. M., \& Urcuioli, P. J. (2002). Stimulus control topographies and tests of symetry in pigeons. Journal of the Experimental Analysis of Behavior, 78, 467-495.

Manabe, K., Kawashima, T., \& Staddon, J. E. R. (1995). Differential vocalization in budgerigars: Towards an experimental analysis of naming. Journal of the Experimental Analysis of Behavior, 63, 111-126.

McIlvane, W. J., \& Dube, W. V. (2003). Stimulus control topography coherence theory: Foundations and extensions. The Behavior Analyst, 26, 195-213

McIlvane, W. J., Kledaras, J. B., Callahan, T. C., \& Dube, W. V. (2002). High-probability stimulus control topographies with delayed $S+$ onset in a simultaneous discrimination procedure. Journal of the Experimental Analysis of Behavior, 77, 189-198.

McIlvane, W. J., Kledaras, J. B., Munson, L. C., King, K. A., de Rose, J. C., \& Stoddard, L. T. (1987). Controlling relations in conditional discrimination and matching by exclusion. Journal of Experimental Analysis of Behavior, 48, 187-208. 
McIlvane, W. J., Serna, R. W., Dube, W. V., \& Stromer, R. (2000). Stimulus control topography coherence and stimulus equivalence: Reconciling test outcomes with theory. In J. Leslie e D. E. Blackman (Orgs.). Issues in experimental and applied analysis of human behavior. Reno: Context Press.

McIntire, K. D., Cleary, J., \& Thompson, T. (1987). Conditional relations by monkeys: Reflexivity, symmetry, and transitivity. Journal of the Experimental Analysis of Behavior, 47, 279-285.

Nevin, J. A. (1969). Signal detection theory and operant behavior: A review of David M. Green and John A. Swets' Signal Detection Theory and Psychophysics. Journal of the Experimental Analysis of Behavior, 12, 475480.

Ray, B. A. (1969). Selective attention: The effects of combining stimuli which control incompatible behavior. Journal of the Experimental Analysis of Behavior, 12, 539550.

Schusterman, R. J., \& Kastak, D. (1993). A california sea lion (Zalophus californianus) is capable of forming equivalence relations. The Psychological Record, 43, 823844.

Sidman, M., \& Tailby, W. (1982). Conditional discrimination vs. matching-to-sample: An expansion of testing paradigm. Journal of the Experimental Analysis of Behavior, 37, 5-22.

Sidman, M. (1971). Reading and auditory-visual equivalences. Journal of Speech and Hearing Research, 14, 5-13.

Sidman, M. (1987). Two choices are not enough. Behavior Analysis, 22, 11-18.
Sidman, M. (1990). Equivalence relations: Where do they come from? In. H. Lejuene \& D. Blackman (Orgs.). Behavior analysis in theory and practice: Contributions and controversies (pp. 93-114). Hillsdale, NJ: Erlbaum.

Sidman, M. (1994). Equivalence relations and behavior: A research story. Boston, MA: Authors Cooperative, Inc., Publishers.

Sidman, M. (2000). Equivalence relations and the reiforcement contingency. Journal of the Experimental Analysis of Behavior, 74, 127-46.

Sidman, M., Rauzin, R., Lazar, R., Cunningham, S., Tailby, W., \& Carrigan, P. (1982). A search for symmetry in the conditional discrimination of rhesus monkeys, baboons, and children. Journal of the Experimental Analysis of Behavior, 37, 23-44.

Tomonaga, M., Matsuzawa, T., Fujita, K., \& Yamamoto, J. (1991). Emergence of symmetry in a visual conditional discrimination by chimpanzees (Pan troglodytes). Psychological Reports, 68, 51-60.

Urcuioli, P. J., \& Zental, T. R. (1993). A test of comparison-stimulus substutability following one-tomany matching by pigeons. The Psychological Record, 43, 745-759.

Vaughan, W. (1988). Formation of equivalence sets in pigeons. Journal of the Experimental Psychology: Animal Behavior Processes, 14, 36-42.

Zygmont, D. M., Lazar, R. M., Dube, W. V., \& Mcllvane, W. J. (1992). Teaching arbitrary matching via sample stimulus-control shaping to young children and mentally retarded individuals: A methodological note. Journal of the Experimental Analysis of Behavior, 57, 109117. 